\title{
Regional Drought Monitoring and Analyzing Using MODIS Data - A Case Study in Yunnan Province
}

\author{
Guoyin Cai, Mingyi Du, and Yang Liu \\ School of Geomatics and Urban Information, Beijing University of Civil Engineering and \\ Architecture, No. 1, Zhanlanguan Road, Xicheng District, Beijing 100044, P.R. China \\ cgyinabucea.edu.cn
}

\begin{abstract}
Yunnan suffered from a severe drought from Winter, 2009 to Spring, 2010. Compared with traditional methods for drought monitoring, remote sensing (RS) based methods has the merits of almost real-time and large covering area. In this paper, vegetation water supply index (VWSI) was used to detect the drought condition utilizing Moderate Resolution Imaging Spectroradiometer (MODIS) data in Yunnan province on 7 March, 2010. Merits and pitfalls of this method were analyzed according to the comparison between the RS detected drought and the real drought status developed by the National Meteorological Bureau. Our results showed that VWSI was apt to agriculture fields with dense vegetation covered areas. This means that this method can be used to detect the drought in a regional area.
\end{abstract}

Keywords: Drought, VWSI index, MODIS, Yunnan Province.

\section{Introduction}

Drought refers to the abnormal water shortage resulted from the imbalance of water budget. When it changes to threaten human's daily life and living conditions, drought becomes drought disaster. An extremely drought was occurred in Yunnan Province from Winter 2009 to Spring 2010. This is the biggest drought occurred in Yunnan province in a century, causing an extremely shortage of water for agriculture, forestry, human beings and living stocks, and a huge loss in local and national economy. So drought monitoring is very important for the local government to make some measurements to reduce losses and water supplying. Classic drought monitoring approaches were based on the point-based data predominantly using meteorological observations and regional drought records, such as the Palmer drought severity index (PDSI) (Palmer 1965)[1], the crop moisture index (Palmer 1968)[2], the surface water supply index (Shafer and Dezman 1982)[3] and the standardized precipitation index (SPI) (McKee et al. 1993, 1995)[4,5]. However, because the meteorological observations are scarcely distributed and most of the underlying surfaces are not homogeneous, so the water conditions obtained from meteorological observations are not representative to the regional conditions (Cai et al. 2007)[6]. Because of the characteristics of almost real time and large covering of areas, remote sensing technology has become the most promising tool for large scale monitoring of drought (Liu and 
Kogan, 1996)[7], furthermore, remote sensing data are continuous datasets consistently available and can be used to detect the onset of drought, its duration and magnitude (Thiruvengadachari and Gopalkrishna, 1993)[8]. Generally speaking, there are three different ways for drought monitoring using remote sensing. 1), detecting soil moisture using remote sensing, and then the drought condition is derived using relationship between soil moisture and drought; 2) Since vegetation developing status was found to be sensitive to water stress, vegetation index is regarded as the indicator of the drought condition, such as Normalized Difference Vegetation Index (NDVI)[9], Atmospherically Resistant Vegetation Index (ARVI)[10], Soil Adjusted Vegetation Index (SAVI)[11] and Enhanced Vegetation Index (EVI)[12]. If vegetation index is calculated from satellite images, drought condition is acquired based on the empirical relationship between vegetation index and drought; 3) like vegetation index, land surface temperature (LST) is also sensitive to drought phenomenon. LST is a good indicator of the energy balance at the earth's surface processes on regional and global scales (Wan et al. 2004)[13]. The main drawback using LST to detect drought is that it is quite difficult to normalize the variation of daily meteorological conditions such as net radiation, air temperature, wind speed, humidity, which affect daytime thermal measurements (McVicar and Jupp 1998[14]). So the vegetation index and LST are combined to evaluate drought status in most cases.

Because the soil moisture derived from satellite images is not too accuracy to detect the regional drought, plus, the different indexes are easy to obtain with a higher spatial and temporal resolution, so the NDVI has been used extensively for drought monitoring in recent years ( $\mathrm{Ji}$ and Peters 2003[15], Vicente-Serrano 2006[16]); Chen et al. (1994)[17] developed the anomaly vegetation index (AVI) to study annual vegetation dynamics. Xiao et al. (1995)[18] developed the Water Supplying Vegetation Index (WSVI), which detects drought by combing vegetation information with temperature retrieved from National Oceanic and Atmospheric Administration (NOAA) satellite data. Qin et. Al. (2008)[19] developed a new index called perpendicular drought index (PDI), which is defined as a line segment that is parallel with the soil line and perpendicular to the normal line of line line intersecting the coordinate origin in the two-dimensional scatter plot of red against near infrared (NIR) wavelength reflectance, to evaluate the drought status of northwestern China using TerraModerate Resolution Imaging Spectroradiometer (MODIS) data. Yet the accuracy of drought monitoring by using the NDVI based drought index may be affected by the change of vegetation type and difference in ecosystems. In addition, the NDVI based drought index may be serve as an after-effect indicator of drought since there are time lags between drought occurrence resulting either from soil moisture deficiency or abnormal reduction of railfull for some consecutive time periods and change of the NDVI.

The combination of NDVI and LST has proved better understanding of drought events with their close inter-relations with surface drought status. The ratio of NDVI and LST, also called the temperature-vegetation index (TVX) (Prihodko and Goward, 1997[20]), has been proven to be significantly correlated with crop moisture (Nemani et al, 1993[21]) and soil moisture in most climatic and land cover conditions (Carlson et al., 1994[22]). Results from McVicar and Biencirth (2001) [23] indicated that the composite AVHRR LST/NDVI was a rapid and effective indicator for drought assessment at country or province level. Beside the LST/NDVI approach, the LST 
versus NDVI scatter plot reveals more meaningful information of drought. Based on the "Triangle" space, the vegetation temperature condition index (VTCI) (Wang et al. 2001[24]) and temperature-vegetation dryness index (TVDI) (Sandholt et al. 2002[25]) were developed for assessing soil moisture status. Because NDVI was easily saturated when there were dense vegetation, Han et al. (2006)[26] discussed the relationship between LST and NDVI in the "triangle" space and replaced NDVI with LAI to further interpret the drought condition when NDVI was saturated.

In this paper, VWSI, which is the combination of LST and NDVI, is used to detect the drought in Yunnan province. The aim of our study was to evaluate the drought in Yunnan province using MODIS data and validate the effectiveness of VWSI for drought monitoring in dense vegetation covered area. In what follows, the study area and data source are given, as are the methodology employed in the research. The results and analysis are then presented. The paper closes with preliminary conclusion and perspectives.

\section{Experiment and Methods}

\subsection{Study Area}

Yunnan is a southwestern province in China. It is a Plateau in low latitude with complex landscape. It is controlled by the dry continental monsoon in winter, and the moist ocean winds in summer. There are 7 climate types in Yunnan province, they are northern tropical climate zone, northern subtropical climate zone, middle subtropical climate zone, middle temperate climate zone, southern subtropical climate zone, southern temperate climate zone, and plateau climate zone. The annual precipitation in most parts of Yunnan province is $1100 \mathrm{~mm}$ or so, but distribution of precipitation is uneven in seasons and regions because of influences of different atmospheric circulation in winter and summer. Almost $89-90 \%$ of rainfall concentrated in May to October, especially in June to August in which the precipitation almost accounts to $60 \%$ of the total annual precipitation. The dry season is from December to next years' April when the precipitation occupies $10-20 \%$ even less of the total annual precipitation. This results in the occurrence of spring drought, causing more frequency occurrence of forest fire and influencing the normal growth of crops.

\subsection{Data and Processing}

MODIS is a key instrument aboard the Terra (EOS AM) and Aqua (EOS PM) satellites. Terra's orbit around the Earth is timed so that it passes from north to south across the equator in the morning, while Aqua passes south to north over the equator in the afternoon. MODIS has a viewing swath width of $2,330 \mathrm{~km}$ and views the entire surface of the Earth every one to two days. Its detectors measure 36 spectral bands between 0.405 and $14.385 \mu \mathrm{m}$, and it acquires data at three spatial resolutions -- $250 \mathrm{~m}, 500 \mathrm{~m}$, and $1,000 \mathrm{~m}$. These data will improve our understanding of global dynamics and processes occurring on the land, in the oceans, and in the lower atmosphere. MODIS is playing a vital role in the development of validated, global, interactive Earth system models able to predict global change accurately enough to assist policy makers in making sound decisions concerning the protection of our environment. 
MODIS Terra level 1B data on 4 March 2010 was downloaded from official MODIS website to detect the drought status in Yunnan province. Before using MODIS data to calculate some parameters, several pre-processing steps are needed as: (1) geometric calibration: MODIS 1B datasets provide us with the latitude and longitude dataset, which can be used to correct the MODIS 1B data. First, the latitude and longitude should be resized to the same size as reflective dataset by bilinear algorithm, then the resized latitude and longitude are used to build a geographic lookup table (GLT) file which contains map locations for every pixel of the image it is associated with, finally, the image is geo-referenced using the established GLT; (2) image cropping: because our study area is only part of the georeferenced one swath of MODIS data, so the image cropping should be applied. First, the vector data of Yunnan province is overlaid to the image, then the region of interest (ROI) covering Yunnan province is selected, and finally the selected ROI is cropped; (3) cloud screen :to remove the cloud influences, cloud screening should be performed in the study area. The near infrared reflection method is used in this paper (Leprieur et al., 1996)[27]; (4) water body removing: because we focus on the land surface, so we removed the water body before calculating NDVI and LST to monitor the drought. Because there is a big difference between water body and land in band 5 of MODIS data, so the threshold method is used in our research to remove the water body.

\subsection{Methodology}

VWSI is defined as:

$$
V W S I=\frac{N D V I}{L S T}
$$

When crops suffer from drought, their leaf apertures partly close to reduce the loss of water, causing the temperature of the leaf surface to increase. The more severe the drought is, the higher the temperature of the leaf surface. At the same time, the growth of crops is affected by drought, resulting in a reduction of the leaf area index (LAI). Leaves will also wither under a high air temperature. All of these factors may result in a reduction of the NDVI. The result is an index, the WSVI, showing the influence of drought on agriculture, and maps of summer drought over large areas. Using this method, vegetation growth can be closely monitored and the regional effects of summer drought can be closely monitored.

NDVI is a simple numerical indicator that can be used to analyze remote sensing measurements, typically but not necessarily from a space platform, and assess whether the target being observed contains live green vegetation or not. The NDVI is calculated as a ratio between measured reflectivity in the red and near infrared portions of the electromagnetic spectrum. These two spectral bands are chosen because they are most affected by the absorption of chlorophyll in leafy green vegetation and by the density of green vegetation on the surface. Also, in red and near-infrared bands, the contrast between vegetation and soil is at a maximum.

The NDVI transformation is computed as the ratio of the measured intensities in the red (R) and near infrared (NIR) spectral bands using the following formula:

$$
N D V I=\frac{N I R-\text { red }}{N I R+\text { red }}
$$


The resulting index value is sensitive to the presence of vegetation on the Earth's land surface and can be used to address issues of vegetation type, amount, and condition. Many satellites have sensors that measure the red and near-infrared spectral bands, and many variations on the NDVI exist. MODIS is a 36 channel radiometer with channels in the red (channel 1) and near infrared (channel 2) potion of the spectrum. The MODIS NDVI is created using data from these channels in the following manner:

$$
N D V I=\frac{\text { channel }_{2}-\text { channel }_{1}}{\text { channel }_{2}+\text { channel }_{1}}
$$

LST is derived using the local split-window algorithm, a common and popular way to calculate the LST. The philosophy of our iterative self-consistent approach for land surface temperature determination is based on the fact that the actual land surface temperatures are the same for all thermal bands (Xue et al., 2005)[28]. Based on the local split-window method (Becker and Li, 1990[29]), for three thermal bands of MODIS data, we have

$$
T_{s, 1}=A_{0}+P_{1} \frac{T_{1}+T_{2}}{2}+M_{1} \frac{T_{1}-T_{2}}{2}
$$

and

$$
T_{s, 2}=A_{0}+P_{2} \frac{T_{1}+T_{3}}{2}+M_{2} \frac{T_{1}-T_{3}}{2}
$$

where $T_{1}, T_{2}$ and $T_{3}$ are the brightness temperature for 1 st, 2 nd, and 3rd thermal infrared band respectively. $A_{0}$ is a constant, $P_{1}, P_{2}, M_{1}$ and $M_{2}$ can be expressed as functions of emissivities $\left(\varepsilon_{1}, \varepsilon_{2}, \varepsilon_{3}\right)$ in these four thermal infrared bands. Theoretically, $\mathrm{T}_{\mathrm{s}, 1}$ is equal to $\mathrm{T}_{\mathrm{s}, 2}$. Because of the effects of emissivity and atmosphere, the temperatures derived from equations 4 and 5 are different in practice. MODIS has 5 thermal bands, 29,30,31,32, and 33. Because band 30 has too much ozone absorption to use, band 33 located in the edge of thermal bands, so bands 29,31,32 are used to constitute two groups to determine the LST. In order to calculate emissivities and LSTs, the study area was classified as several land cover types using the above mentioned method. Each land cover type was regarded as one window. To enable comparisons to be made for the two sets of satellite data, all pixels $(\mathrm{Pm})$ in the window were chosen from each set of satellite data. The detailed description of this method is in Xue et al. (2005).

\section{Results}

After NDVI and LST are calculated, VWSI is computed using band math in software ENVI. Based on the drought levels, that is, extreme drought, severe drought, moderate drought, slight drought and normal, are obtained by means of density slice.

Figure 1 is the NDVI which generally shows the growth of vegetation in Yunnan province. Figure 1 indicates that the vegetation growth developed not well in most parts of our study areas in color yellow and cyan, except small parts in the southern and western parts in color purple. Areas in white are covered by cloud. This means that the vegetation growth in Yunnan Province is not good at this time when it is developed well in normal years. 


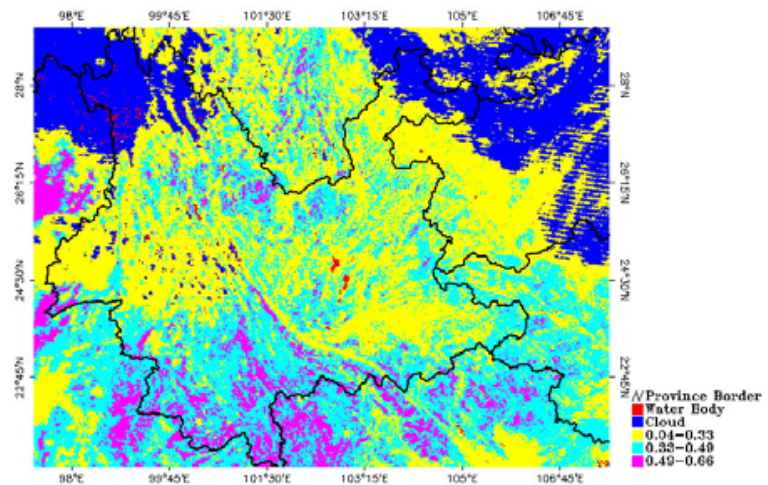

Fig. 1. NDVI derived from MODIS data in the Yunnan Province on 7 March, 2010

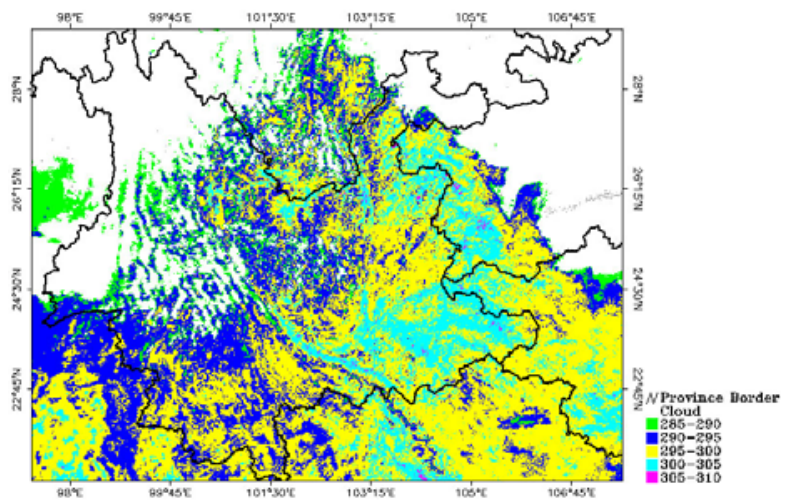

Fig. 2. LST derived from MODIS data in the Yunnan Province on 7 March, 2010

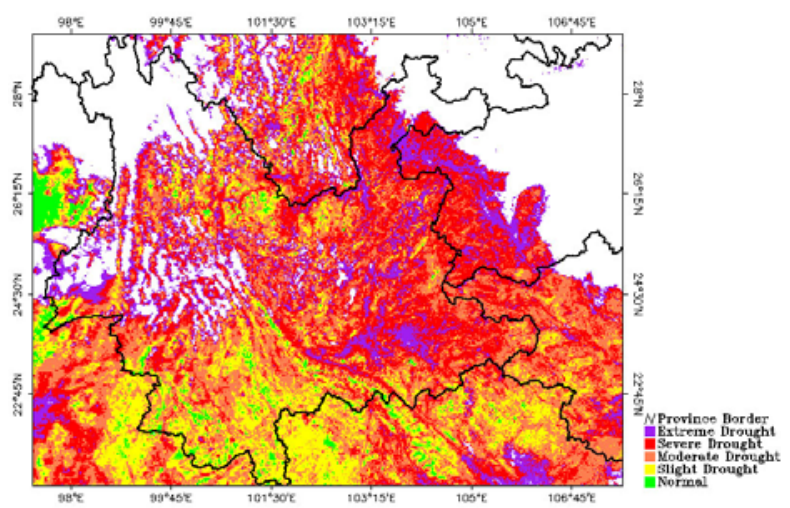

Fig. 3. LST derived from MODIS data in the Yunnan Province on 7 March, 2010 
Figure 2 is the LST in the study area. The temperatures in our study area are from 290K-310K. Generally speaking, temperatures in the northern and western regions are lower than that in eastern regions. This indicates that, compared with western areas, there are severe drought in the eastern regions.

Figure 3 is the VWSI from which we can see that most of the areas are in drought, especially in the eastern part of Yunnan Province. This is in a consistency with the real drought status monitored and issued by the National Meteorological Bureau.

\section{Conclusions and Perspectives}

Our research shows that VWSI index can be used to obtain the drought information in Yuannan province. This indicates that VWSI can be adopted to monitor drought in a regional scale from satellite images. There are some issues should be taken into consideration when using VWSI. First, it is difficult to collect the satellite images in a cloud-free day in a regional scale, even using MODIS data with $1 \mathrm{~km}$ spatial resolution; Second, the underlying surfaces should be covered mostly by vegetation when using VWSI index, otherwise, it should be replaced by other indexes such as soil moisture or thermal inertia; third, VWSI is a relative value with no real meaning, the level of drought based on VWSI should be determined by empirical, this influences the accuracy of the monitoring results. According to those issues, our further research should focus on using some satellite images in microwave bands without influencing of cloud. Furthermore, a drought monitoring system should be developed to detect the drought status and give some prediction for the future possible drought. This might be helpful for the local government and farmers to make some measurements to decreasing losses.

Acknowledgments. The Publication is supported by Beijing Natural Science Foundation under Grant No.4102018, and by Beijing Municipal Organizing Department under Grant No. 070800905, and is also supported partly by Jurisdiction of Beijing Municipality under Grant No. PHR200906138, PHR200907127 and PHR 20070101.

\section{References}

1. Palmer, W.C.: Meteorological Drought. Research Paper No.45, US Department of Commerce Weather Bureau, Wshington, DC (1965)

2. Palme, W.C.: Keeping track of crop moisture conditions, nationwide: the Crop Moisture Index. Weatherwise 21, 156-161 (1968)

3. Shafer, B.A., Ezman, L.E.: Development of a surface water supply index (SWSI) to access the severity of drought conditions in snowpack runoff areas. In: Proceedings of the 50th Annual Western Snow Conference, Colorado State University, pp. 164-175 (1982)

4. McKee, T.B., Doeskin, N.J., Kleist, J.: The relationship of drought frequency and duration to time scales. In: Proceedings of 8th Conference on Applied Climatology, Boston, pp. 179-184 (1993)

5. McKee, T.B., Doeskin, N.J., Kleist, J.: Drought monitoring with multiple time scales. In: Proceeding of 9th Conference on Applied Climatology, Boston, pp. 233-236 (1995) 
6. Cai, G.Y., Xue, Y., Hu, Y.C.: Soil Moisture Retrieval from MODIS data in Northern China Plain using thermal inertia Model. International Journal of Remote Sensing 16, 3567-3581 (2007)

7. Liu, W., Kogen, F.N.: Monitoring regional drought using the vegetation condition index. International Journal of Remote Sensing 17, 2761-2782 (1996)

8. Thiruvengadachari, S., Gopalkrishna, H.R.: An integrated PC environment for assessment of drought. International Journal of Remote Sensing 14, 3201-3208 (1993)

9. Rouse, J.W., Haas, R.H., Schell, J.A.: Monitoring the Vernal Advancements and Retrogradation, Texas, Texas A \& M University (1974)

10. Kaufman, Y.J., Tanre, D.: Atmospherically Resistant Vegetation Index (ARVI) for EOSMODIS. IEEE Transaction on Geoscience Remote Sensing 30, 261-270 (1992)

11. Huete, A.R.: A Soil Adjusted Vegetation Index (SAVI). Remote Sensing of Environment 25, 295-309 (1988)

12. Liu, H.Q., Huete, A.R.: A feedback based Modification of the NDVI to Minimize Canopy Background and Atmospheric Noise. IEEE Transaction on Geoscience and Remote Sensing 33, 457-465 (1995)

13. Wan, Z., Wang, P., Li, X.: Using MODIS land surface temperature and normalized difference vegetation index products for monitoring drought in the southern great plains, USA. International Journal of Remote Sensing 25, 61-72 (2004)

14. Mcvicar, T.R., Jupp, D.L.B.: The current and potential operational uses of remote sensing to aid decisions on drought exceptional circumstances in Australia: a review. Agriculture System 3, 399-468 (1998)

15. Song, X., Saito, G., Kodama, M.: Early detection system of drought in East Asia using NDVI from NOAA AVHRR data. International Journal of Remote Sensing 25, 3105-3111 (2004)

16. Vicente-Serrano, S., Cuadrat-prats, J.M., Romo, A.: Early prediction of crop productivity using drought indices at different time scales and remote sensing data: application in the Ebro valley (north east Spain). International Journal of Remote Sensing 27, 511-518 (2006)

17. Chen, W., Xiao, Q., Sheng, Y.: Application of the anomaly vegetation index to monitoring heavy drought in 1992. Remote Sensing of Environment 9, 106-112 (1994) (in Chinese)

18. Kogan, F.N.: Application of vegetation index and brightness temperature for drought detection. Advances in Space Research 15, 91-100 (1995)

19. Qin, Q., Chulam, A., Zhu, L.: Evaluation of MODIS derived perpendicular drought index for estimation of surface dryness over northwestern China. International Journal of Remote Sensing 7, 1983-1995 (2008)

20. Prihodko, L., Goward, S.N.: Estimation of air temperature from remotely sensed surface observations. Remote Sensing of Environment 60, 335-346 (1997)

21. Nemani, R., Price, L.L., Running, S.W.: Developing satellite derived estimates of surface moisture status. Journal of Applied Meteorology 32, 548-557 (1993)

22. Carlson, T.N., Gillies, R.R., Perry, E.M.: A method to make use thermal infrared temperature and NDVI management to infer surface soil water content and fractional vegetation cover. Remote Sensing Reviews 9, 161-173 (1994)

23. McVicar, T.R., Biencirth, P.N.: Rapidly assessing the 1997 drought in Papua New Guinea using composite AVHRB imagery. International Journal of Remote Sensing 22, 2109$2128(2001)$

24. Wang, P., Gong, J., Li, X.: Vegetation-Temperature Condition Index and its application for drought monitoring. Geomatics and Information Science of Wuhan University 26, 412 418 (2001) (in Chinese) 
25. Sandholt, I., Rasmussen, K., Andersen, J.: A simple interpretation of the surface temperature/vegetation index space for assessment of surface moisture status. Remote Sensing of Environment 79, 213-224 (2002)

26. Han, L., Wang, P., Yang, H.: Study on NDVI-Ts space by combining LAI and evapotranspiration. Science in China Series D 49, 747-754 (2006)

27. Leprieur, C., Kerr, Y.H., Richon, J.M.: Critical assessment of vegetation indices from AVHRR in a semiarid environment. International Journal of Remote Sensing 13, 25492563 (1996)

28. Xue, Y., Cai, G.Y., Guan, Y.N.: Iterative self-consistent approach for earth surface temperature determination. International Journal of Remote Sensing 26, 185-192 (2005)

29. Becker, E., Li, Z.L.: Towards a local spilt window method over land surface. International Journal of Remote Sensing 11, 369-394 (1990) 